\title{
La enseñanza de la lectura y la escritura académicas mediante programas a lo largo del curriculum universitario: opción teórica, didáctica y de gestión
} O ensino da leitura e da escrita acadêmicas mediante programas ao longo do currículo universitário: opção teórica, didática e de gestão

Teaching of academic reading and writing through programs across the university curriculum: theoretical, pedagogical and administrative choices

Estela Inés MOYANO

(Universidad de Flores - Programa de Lectura y Escritura Académicas (PROLEA-UFLO) -

Ciudad Autónoma de Buenos Aires - Argentina)

\section{RESUMEN}

En este trabajo se aborda el desarrollo de la lectura y la escritura académicas y profesionales en el nivel universitario. En primer lugar, se justifica su enseñanza en Argentina, atendiendo al sistema universitario local, las características de los estudiantes y las del discurso que deben abordar en ese nivel, lo que vuelve generalizable el planteo a otros contextos. Luego, se presenta como opción pedagógica la instalación de programas de lectura y escritura a lo largo de la carrera universitaria y sus requerimientos teóricos, metodológicos y de gestión: una teoría del aprendizaje, una teoría de contexto y lenguaje, una propuesta didáctica y una de gestión académica. Se presenta la teoría del aprendizaje a través del lenguaje de la Lingüistica Sistémico Funcional (LSF); como teoría del lenguaje, se propone la teoría de género y discurso en el marco de la LSF; como opción didáctica, la adaptación de la pedagogía basada en géneros formulada por la Escuela de Sydney y su adecuación al contexto universitario argentino. Luego, se explicitan las características de diseño de un programa, los problemas que presenta para su gestión y propuestas 
para su superación. Finalmente, se enuncian algunos resultados de su implementación.

Palabras clave: escritura en el nivel superior; programas de escritura; teoría de género y discurso; Lingüística Sistémico-Funcional; didáctica basada en géneros.

\section{RESUMO}

Neste trabalho aborda-se o desenvolvimento da leitura e da escrita acadêmica e profissional em nível universitário. Em primeiro lugar, se justifica seu ensino na Argentina em atendimento ao sistema universitário local e se expõem as características dos estudantes e do discurso a ser abordado nesse nível, o que torna generalizável sua proposição em outros contextos. Em seguida, se apresenta como opção pedagógica a implantação de um programa e de leitura e escrita ao longo da carreira universitária e explicitam-se suas características da organização. Na sequência, se enunciam suas exigências teóricas, metodológicas e de gestão: uma teoria de aprendizagem, uma teoria de contexto e linguagem, uma proposta didática e uma de gestão acadêmica. Apresenta-se a teoria da aprendizagem por meio da linguagem da Linguística SistêmicoFuncional (LSF); como teoria de linguagem, propõe-se a teoria de gênero e discurso no marco da LSF; como opção didática, a adaptação da pedagogia baseada em gêneros idealizada pela Escola de Sydney e sua adequação ao contexto universitário argentino. Logo, explicitam-se os problemas que surgem em sua gestão e propostas para sua superação. Por fim, se enunciam alguns resultados de sua implementação.

Palavras-chave: escrita no nivel superior; programas de escrita; teoria de gênero e discurso; Linguística Sistêmico-Funcional; didática baseada em gêneros.

\section{ABSTRACT}

This paper deals with the development of academic and professional reading and writing at the tertiary level. First, the paper argues for the teaching of reading and writing in Argentina, considering the specific tertiary context, the features of its students and the characteristics of the discourse they need to approach at this level. This consideration enables the proposal of generalizations suitable for other contexts. Then, the paper introduces the pedagogical choice of developing reading and 
writing programs across the tertiary curriculum, as well as the theoretical, pedagogical and administrative requirements for this choice. Thus, the paper presents a theory of learning, a theory of language and context, and a pedagogical and academic program administration proposal. The theory of learning considered corresponds to Systemic Functional Linguistics (SFL), which understands learning as a process enacted through language; as a theory of language, the paper proposes the model of genre and discourse proposed within the framework of SFL; as a pedagogical choice, an adaptation of genre-based pedagogy developed by the Sydney School is introduced and its adaptations to the Argentinian university context are presented. Finally, the features of a program design are made explicit, as well as some problems arising from its administration and proposals for its solution. The paper ends with a discussion of some of the results emerging from the implementation of the program.

Key-words: writing at tertiary education; writing programs; theory of genre and discourse; Systemic functional linguistics; genre-based pedagogy.

\section{Introducción}

En la etapa de ingreso a la Universidad, los estudiantes se integran a un nuevo contexto educativo, con prácticas muy diferentes a las que les resultan conocidas, dada su experiencia anterior. En ese contexto, tienen que abordar textos de ámbitos desconocidos para ellos, como los propios del académico y el profesional, con propósitos, estructuras esquemáticas y recursos lingüísticos específicos, ajenos a su conocimiento previo.

Tener en cuenta esta situación puede conducir a dejar de costado ciertos argumentos generalizados en las instituciones universitarias para justificar la creación de espacios dedicados a la enseñanza de la lectura y la escritura en el nivel superior. En principio, no se trata de que haya que resolver ineficiencias de los niveles educativos anteriores, pues los textos que circulan en la universidad son de naturaleza diferente de los que se leen y escriben en la escuela secundaria. Tampoco se trata de deficiencias o desidia de los estudiantes que no se dedican lo suficiente al estudio, sino de que no han recibido aún la formación necesaria para abordar textos con las características antes mencionadas. 
Esto implica la necesidad de modificar la mirada sobre el problema de la enseñanza de la lectura y la escritura en el nivel universitario. Es necesario dejar de pensarlo como un problema ajeno, como una carga extra a incorporar al curriculum o a asignar a los profesores de las materias específicas a una carrera. Se trata, en realidad, de un problema propio del nivel, que hay que abordar con el fin de contribuir al mejor desarrollo del aprendizaje de contenido de los estudiantes, para prepararlos para un mejor desempeño en la universidad así como para su futura actuación como profesionales. No se trata solo de considerarlo como desarrollo de habilidades específicas de lectura y escritura de textos disciplinares y profesionales, sino también como recurso para el aprendizaje de contenidos y para la producción de nuevas conceptualizaciones a medida que se vaya avanzando en la enseñanza de estas habilidades y de los recursos lingüísticos y discursivos que demandan los textos a abordar. Asimismo, se trata de entender este proceso de aprendizaje como la reflexión sobre las nuevas prácticas sociales que deben ser realizadas a través del uso del lenguaje en los contextos a los que los estudiantes ingresan.

En este trabajo se abordará esta cuestión, teniendo en cuenta una serie de factores que justifican la atención de esta necesidad. Si bien se considera el contexto universitario argentino, se procurará mostrar que se trata de un fenómeno generalizable. Asimismo, se propondrá como recurso de gestión pedagógica la instalación de programas de lectura y escritura a lo largo del curriculum universitario tomando como caso el Programa de Lectura y Escritura Académicas (PROLEA) que se encuentra en proceso de consolidación en la Universidad de Flores (UFLO), en Argentina. Se presentará brevemente su diseño, $\mathrm{y}$, especialmente, se señalarán los requerimientos teórico-metodológicos que se entienden como imprescindibles desde la perspectiva que se adopta como marco general. Se abordarán también los problemas que presentan programas con estas características y una propuesta de solución. Finalmente, se mencionarán algunos resultados de esta experiencia.

\section{El abordaje de la lectura y la escritura en la universidad}

Ya en 1994, José Luis Coraggio planteaba dos causas para lo 
primer lugar, las escasas habilidades de los estudiantes para la lectura de textos - que diversos investigadores habían señalado o lo hicieron posteriormente (De Lelia y Ezcurra 1994; Ezcurra 1995; Ameijide et al 2000; Pereira y di Stefano 2001; UnLu. 2001; Zalba 2002; Cubo de Severino 2002; Uslenghi et al 2002; Padilla 2007, Moyano 2003a; 2007) -, además de sus limitadas habilidades para la resolución de problemas; en segundo término, las interrupciones frecuentes de los estudios, seguidos de la deserción de los programas académicos. No es imposible pensar que estos factores estén relacionados, dado que las dificultades para el acceso a los contenidos a aprender a partir de la lectura de la bibliografía puede ser una causa de expulsión del nivel superior para quienes tengan dificultades de comprensión del lenguaje científico. Es cierto que existen posibilidades de estudiar a partir de apuntes, es decir, de resúmenes de contenidos elaborados por los mismos docentes u otros estudiantes, pero este recurso limita a los estudiantes en su aprendizaje.

En un contexto como el argentino, en el que el ingreso a las universidades está garantizado para estudiantes de diferentes procedencias sociales, cabe considerar que, en consecuencia, la población universitaria estará conformada de manera heterogénea. Esta situación, en términos de Basil Bernstein (1990), implica que se podrá observar una distribución desigual de los recursos lingüísticos entre estudiantes, en desmedro de los que provengan de clases de menor capital cultural y etnias no centrales. En la Argentina, sin embargo, no se puede atribuir exclusivamente el problema a una cuestión de clase, pues existen organizaciones sociales que se ocupan especialmente de la formación en lectura y escritura de niños y jóvenes de clases menos favorecidas. Aun así, se puede postular que existe una brecha entre estudiantes que han recibido una buena formación en sus hogares y en buenas escuelas y otros que provienen de una escolarización menos ventajosa, que los deja fuera de la zona de desarrollo próximo (Vigotsky, 1998) en relación con los nuevos aprendizajes exigibles en lectura y escritura académicas. Si la universidad no se ocupa de achicar esa brecha, dejará fuera de su esfera a los estudiantes con menos recursos.

En efecto, Ana María Ezcurra (2011) sostiene que las universidades son responsables de asegurar la permanencia y graduación de los 
sujetos que ocupan sus aulas a través de la selección de los contenidos $y$ habilidades que debe enseñar con ese fin. La autora acuña el concepto de "inclusión excluyente" para referirse a la aparente democratización de los estudios superiores que resulta de la gratuidad de la enseñanza pública argentina si no se logra la graduación de todos los estudiantes.

Una de las habilidades cuyo abordaje debe encarar la universidad es, precisamente, la que permite operar con los contenidos disciplinares a través de la lectura y la escritura de textos académicos y profesionales. La lectura de la bibliografía, como resulta obvio decir, permitirá un acceso más completo al conocimiento que necesitan abordar los estudiantes para aprobar una asignatura que el que ofrece la explicación del profesor en sus clases magistrales y la simplificación de esos contenidos a través de notas de otros estudiantes o de documentos preparados por los mismos profesores. La escritura, como lo han señalado autores como Bereiter y Scardamaglia (1987), permite reelaborar lo aprendido transformando el conocimiento, lo que implica un mayor aprendizaje. En efecto, si se acepta que el mayor dominio de los recursos lingüísticos favorece la conceptualización (Vigotsky 1998; Halliday 1993a), se podrá concluir que el operar con la escritura, incorporando nuevos recursos y más sofisticados, como los característicos del discurso de las ciencias (Halliday \& Martin 1993; Martin \& Veel 1998; Hood \& Martin 2005; Korner, McInnes \& Rose 2007; Veel 2007; Wignell 2007), necesariamente contribuirá a la construcción de nuevos significados en el campo disciplinar de que se trate, es decir, a la producción de nuevos aprendizajes.

Por otra parte, si se entienden las nuevas prácticas sociales que los estudiantes deben llevar a cabo a través del lenguaje como géneros y macrogéneros, siguiendo los conceptos elaborados por Jim Martin (Martin 1992; 2001; 2014; Martin \& Rose 2008; Martin 1994; Eggins \& Martin 2003), resulta relevante también encarar la enseñanza de la estructuración de los textos a abordar, a fin de que los estudiantes puedan operar mejor con el lenguaje entendido como actividad social, produciendo textos que, en su despliegue, logren el propósito perseguido.

Es importante señalar que la "inclusión excluyente" (Ezcurra, 2011) parece darse no solamente en universidades libres de arancelamiento, 
sino en otras aranceladas, tanto en la Argentina como en otros países. Y esto es así, posiblemente, debido no solo a la posibilidad de que coexistan poblaciones heterogéneas, sino a la novedad que, como se señaló en la Introducción, representan las prácticas discursivas que son necesarias en el nivel superior, tanto para acceder a los contenidos de las diferentes disciplinas que se ponen en juego en distintas carreras como a las que se perfilan como necesarias en el ejercicio de las profesiones para las que los estudiantes se forman. Es cierto, si se tiene en cuenta lo señalado por Bernstein (1990), que habrá diferentes necesidades en distintos grupos de estudiantes, que algunos estarán mejor preparados que otros. Sin embargo, la necesidad de formación en estos campos alcanza a todos.

Es posible sintetizar hasta aquí qué necesitan los estudiantes, cualquiera sea su procedencia social, para avanzar hasta la graduación y prepararse para el estadio siguiente, el de la práctica profesional: necesitan de una práctica orientada de lectura y escritura para aprender tanto los contenidos disciplinares como las prácticas académicas y profesionales. Las claves para lograr estos aprendizajes están en el abordaje del contexto social en que los textos a estudiar funcionan, su propósito, su estructura esquemática y las características relevantes del lenguaje utilizado para la producción de significado.

\section{Un programa de lectura y escritura académicas y profesionales a lo largo del curriculum}

Como recurso para abordar la enseñanza de las habilidades requeridas por los estudiantes universitarios en lectura y escritura académicas y profesionales, este trabajo propone, como opción pedagógica, la instalación de programas que tengan como objetivo atender este problema a lo largo de las carreras universitarias. Esto implica que los estudiantes experimenten reiteradas instancias de trabajo con este tipo de discurso vinculadas a diversas asignaturas y en relación con los contenidos de las disciplinas involucradas. Esto se logra con un diseño que contemple la integración de la enseñanza de estas habilidades, con la participación de docentes de Letras que integran el programa en cuestión, en el marco de las actividades de escritura propuestas en las asignaturas. Como ejemplo de este tipo de 
trabajo presentamos el programa que se ha propuesto en la Universidad de Flores, denominado PROLEA-UFLO.

La Universidad de Flores es una universidad privada, de costo accesible, que no toma examen para regular el ingreso de los aspirantes a cursar las carreras que en ella se dictan. En cuanto al perfil de sus estudiantes, son en su mayoría provenientes de la zona de cercanía de la Universidad, ubicada en un barrio de clase media baja. El 75\% de los estudiantes se encuentran en situación laboral activa, y el 50\% de ellos trabajan entre 8 y 12 horas diarias, lo que constituye una limitación para el desarrollo previsto de la cursada de la carrera elegida. Los estudiantes, además, se ubican en un amplio rango de edad, aunque el 65\% tiene entre 17 y 20 años (fuente: Servicio de Orientación al Estudiante de la Universidad).

Un antecedente destacable del PROLEA-UFLO lo constituye el Programa de Desarrollo de Habilidades de Lectura y Escritura Académicas (PRODEAC), establecido en 2005 en la Universidad Nacional de General Sarmiento (UNGS) (Moyano, 2004; 2010; Moyano \& Natale, 2012). Sin embargo, el PROLEA-UFLO presenta características propias, dadas por su contextualización institucional.

En primer lugar, el PROLEA-UFLO se instala desde el primer año de cada carrera y hasta el último, asociándose a una materia por año en cada una de ellas. Pero, además, forma parte de una serie de dispositivos orientados a favorecer el ingreso, la permanencia y la graduación de los estudiantes. Éstos son el Servicio de Orientación al Estudiante (SOE), ubicado al inicio de las carreras; el Programa de Acción Tutorial (PAT), opcional para los estudiantes y que les ofrece asistencia en el momento en que ellos la demanden; y un acompañamiento de la elaboración de tesis con orientación en metodología de la investigación al finalizar los estudios.

En cuanto a su diseño, se sigue la modalidad de asociación entre el Programa y las materias que se seleccionan en acuerdo entre la Coordinación, los Directores de una Carrera y los Decanos de cada Facultad (Moyano \& Giudice 2016a). Esto implica, como ya se señaló para el PRODEAC (Moyano 2009; 2010), el trabajo interdisciplinario entre un profesor de Letras, miembro del Programa y el o los docentes a cargo de las materias de las carreras a las que éste se asocia. De esta manera, no se incluyen talleres de enseñanza de la lectura y la escritura 
dictados por profesores de Letras que desconocen el campo específico de las disciplinas en juego, ni se asigna la responsabilidad completa a los docentes de las materias propias de la carrera. Se trata de un trabajo en el que cada profesional aporta su conocimiento: los docentes de Letras su capacidad para la enseñanza de la lectura y la escritura así como para el análisis de los textos que se trabajan en el aula; los docentes de las materias específicas, el contenido disciplinar en juego y las prácticas profesionales. Se logra, de esta manera, un trabajo que potencia las capacidades de cada profesional en un equipo al que se le asignan una serie de tareas conjuntas que les permiten negociar las características de los textos a enseñar y el trabajo que se llevará a cabo en las aulas (Moyano 2009; 2010; Moyano \& Giudice 2016b).

Como ya ha sido señalado en otras oportunidades, un programa de lectura y escritura a lo largo de toda la carrera universitaria presenta ventajas con respecto a otras modalidades más frecuentemente elegidas por las instituciones universitarias: los talleres ubicados al inicio de los estudios superiores y/o paralelos a las asignaturas específicas de una carrera o la asignación de la responsabilidad de guiar a los estudiantes en el abordaje y la producción de los textos disciplinares a los profesores de las materias específicas a una carrera. Con respecto a los talleres paralelos, a cargo de profesores de Letras, en general se trabaja con textos ajenos a las disciplinas involucradas en las carreras elegidas por los estudiantes. Según Hyland (2002), este tipo de talleres limitan las posibilidades de transferencia de las prácticas aprendidas a otras instancias. En cuanto a la enseñanza de estas habilidades a cargo únicamente de profesores de la materia específica, son cuestionados desde una perspectiva que sostiene la necesidad de reflexionar sobre las características lingüísticas de los textos de especialidad. Este cuestionamiento se debe a que estos docentes no tienen formación en didáctica de la lectura y la escritura ni tampoco en el análisis lingüístico de los textos a trabajar en clase (Moyano 2004).

\section{Requerimientos para un programa de lectura y escritura a lo largo de las carreras universitarias}

La propuesta de programas de lectura y escritura del tipo brevemente descripto en el punto anterior presenta como requerimientos 
fundamentales una teoría de aprendizaje basada en el lenguaje, una opción teórica lingüística que permita sustentar ese aprendizaje, una propuesta didáctica que lo oriente en sus acciones y un diseño de gestión institucional que permita organizar su ejecución. A continuación se enunciarán estas elecciones de manera sintética.

\subsection{Una teoría de aprendizaje a través del lenguaje}

El primer punto a considerar entre los requerimientos de un programa de lectura y escritura a lo largo de una carrera universitaria es una teoría acerca de cómo se producen los aprendizajes de conocimientos especializados. En términos de Bernstein (1999), cómo se efectúan los aprendizajes de "estructuras simbólicas especializadas de conocimiento explícito", ya no relacionadas con las prácticas en contextos cotidianos sino con la producción intelectual de las disciplinas.

A partir de sus propios desarrollos y los de otros estudiosos en el marco de la Lingüística Sistémico-Funcional, Michael Halliday (1993a) esboza una teoría de aprendizaje a través del lenguaje. Halliday considera que el lenguaje es el sistema semiótico más sofisticado producido por los humanos, el recurso para construir una imagen del mundo, el medio para la construcción de conocimiento a través del procesamiento de la experiencia (Halliday 2004; Halliday \& Matthiessen 1999). Así, el lenguaje permite no solo satisfacer las demandas de la comunicación familiar y cotidiana sino, en su evolución, la construcción de conocimiento científico (Halliday 1993b; 1998).

Esta evolución implica no solo la aparición del léxico técnico propio de cada disciplina, lo que en la teoría se conoce como "tecnicalidad", sino, sobre todo, el desarrollo de recursos gramaticales y discursivos especiales para la producción de discurso técnico y abstracto, así como también para la valoración del mundo construido por el discurso y para la construcción de un diálogo entre diferentes posiciones sociales en relación con él (heteroglosia) (Halliday \& Martin 1993; Martin \& Veel 1998; Hood \& Martin 2005; Martin \& White 2005; Veel 2007; Korner, McInnes \& Rose 2007; Wignell 2007). En este sentido, incrementar el desarrollo de las habilidades de producción de significado a través del lenguaje implica acrecentar las capacidades de producir conocimiento: 
el desarrollo del lenguaje es, al mismo tiempo, el desarrollo del aprendizaje (Halliday 1993a).

De ahí que se vuelva necesario, al trabajar con el discurso académico en el aula, la exploración de los textos a fin de reconocer, por un lado, los recursos lingüísticos que se ponen en juego para la construcción de significado, y, por el otro, su estructura, el modo como se desenvuelven en el tiempo del discurso esos significados a fin de lograr los propósitos sociales perseguidos (Martin 1992; 1994; 1997; $2001 ; 2014 ; 2016)$.

Reconocer esos recursos, sistematizarlos, aprenderlos para identificarlos en la lectura y utilizarlos en la escritura o en la producción de textos orales es lo que se entiende como desarrollo del lenguaje. Y esta práctica en el aula permite no solo el desarrollo de habilidades de lectura y escritura en un contexto determinado sino la construcción de conocimiento disciplinar. Así, favorecer el desarrollo del lenguaje, y especialmente de formas del lenguaje propias de esferas institucionales diferentes a las cotidianas o familiares, permitirá el acceso a la construcción de conocimentos diferentes de los del sentido común.

Halliday (2004) también sostiene la existencia de tres tipos de aprendizaje relacionados con el lenguaje que un hablante necesita desarrollar en este proceso. El primero, incluso en el tiempo de la ontogénesis, consiste en "aprender el lenguaje", es decir, el uso de los recursos que éste provee para la interacción en diferentes contextos. El segundo, en "aprender sobre el lenguaje", es decir, la toma de conciencia de los recursos disponibles en un contexto dado para la producción de significados. El tercero se trata de "aprender a través del lenguaje", lo que significa el desarrollo de habilidades para la comprensión y producción de conocimiento mediante el uso del lenguaje.

Dado que el aprendizaje del lenguaje, sobre el lenguaje, y a través del lenguaje se hace en interacción, primero con los cuidadores y luego con los maestros y profesores (Halliday 2004; Painter 1986), es responsabilidad del docente llevar a cabo un trabajo conjunto con los estudiantes para promover esos procesos. Este trabajo, según la propuesta del grupo que recibió el nombre de Escuela de Sydney, se basa en el principio de la "guía a través de la interacción en un contexto de experiencia compartida" (Martin, 2000). Pero sobre esto se dirá más 
al abordar la estrategia didáctica por la que se opta en el modelo de programas propuesto aquí.

\subsection{Teoría de lenguaje en contexto: género y discurso}

El marco teórico lingüístico seleccionado para sustentar un programa de lectura y escritura como el descripto y en ese contexto el aprendizaje del lenguaje, sobre el lenguaje y a través del lenguaje, es la Lingüística Sistémico-Funcional. Esta teoría es entendida por Halliday (2003) como una lingüística aplicable a diferentes propósitos, una lingüística orientada a mostrar cómo se producen significados en los textos, como lo muestran Martin (1992; 1993; 2000; 2009a), Martin $\&$ Rothery (1993) y Martin \& Rose (2007; 2008). En este apartado se presentan sintéticamente los conceptos centrales que resultan de utilidad para el aprendizaje de la lectura y la escritura basada en el lenguaje y los géneros del discurso.

La Lingüística Sistémico-Funcional se propone estudiar cómo usan el lenguaje los hablantes para llevar a cabo la vida en sociedad, qué hace el lenguaje por los humanos y cómo lo hace. La unidad de análisis es el texto en contexto, entendiendo por texto una unidad real de comunicación, siempre en relación con el contexto de uso.

La teoría postula una relación de mutua determinación en términos probabilísticos entre contexto y lenguaje: dado un contexto, es esperable el uso de ciertos recursos del sistema lingüístico; dado un texto con ciertos recursos, es posible inferir su contexto. Más aun, la teoría considera el contexto como intrínseco al texto, es decir, un contexto que se pone de manifiesto en el texto a partir de la selección de recursos del lenguaje para la producción de significados. Estos significados incluyen la construcción de experiencia o de mundo (campo), la de relaciones sociales entre interlocutores (tenor) y la función del lenguaje en la construcción del texto así como su organización y su canal (modo) (Halliday 1982; 2005). Este conjunto de significados es considerado por Martin (1992) como uno de dos estratos del contexto, al que denomina registro.

Un concepto clave para la enseñanza de la lectura y la escritura es el concepto de género, que constituye el estrato contextual más 
abstracto (Martin 1992; Martin \& Rose 2008). En este marco teórico, los géneros se definen como configuraciones recurrentes de significados del registro, que representan o llevan a cabo las prácticas sociales de una cultura dada. Estos significados se manifiestan en los textos - como ya se señaló antes - a través del lenguaje, de manera que pueden ser identificados a partir del reconocimiento de las elecciones de recursos lingüísticos que los construyen.

En una definición más operacional, los géneros son entendidos como proceso social que se realiza en pasos o etapas y está orientado a una meta (Halliday \& Martin, 1993). En este sentido, lo que se pone de relieve es el reconocimiento de una estructura en el texto, que permite, mediante la articulación de partes que se suceden en el despliegue del discurso, lograr el propósito social que lo caracteriza.

En suma, la teoría propone un modelo estratificado de lenguaje en contexto (Martin 1985; 1992; 2014; 2016; Martin \& Rose 2007; 2008) (Figura 1). Por un lado, el contexto está estratificado en género y registro. El género se responsabiliza de la estructuración de los textos y de la combinación de significados de registro seleccionados, que incluyen los que construyen mundo (campo), los que construyen relaciones (tenor) y los que establecen la distancia semiótica en términos de abstracción (modo). Estos significados se realizan a través del lenguaje en tres metafunciones: el campo a través de la metafunción ideacional; el tenor mediante la interpersonal; y el modo, a través de la metafunción textual.

El lenguaje, a su vez, se organiza en tres estratos: el semánticodiscursivo, que tiene por unidad de análisis el texto; el léxicogramatical, cuya unidad es la cláusula; y el fono-gráfico, que permite realizar los significados en unidades de tono y tonicidad o en grafemas, según el texto sea oral o escrito. Así, los significados de las diferentes metafunciones se organizan en sistemas por estratos, que proveen los recursos entre los cuales los hablantes eligen para la producción de significado.

La Figura 1 es la que se utiliza habitualmente para indicar estas relaciones. Así, los diferentes círculos cotangenciales de líneas más gruesas representan el contexto y el lenguaje (respectivamente el superior y el inferior, como se indica mediante las llaves por fuera de 
los círculos). Dado que se trata de dos sistemas semióticos estratificados (Martin 1992; 2014; 2016; Martin \& Rose 2007; 2008), los estratos se grafican mediante círculos cotangenciales de líneas más finas: dos para el contexto (género y regisro) y tres para el lenguaje (semánticadiscursiva, léxico-gramática y fonología o estrato fono-gráfico). Las líneas que cruzan desde el estrato inferior del lenguaje hasta el registro, representan la distinción entre metafunciones del lenguaje y variables del registro.

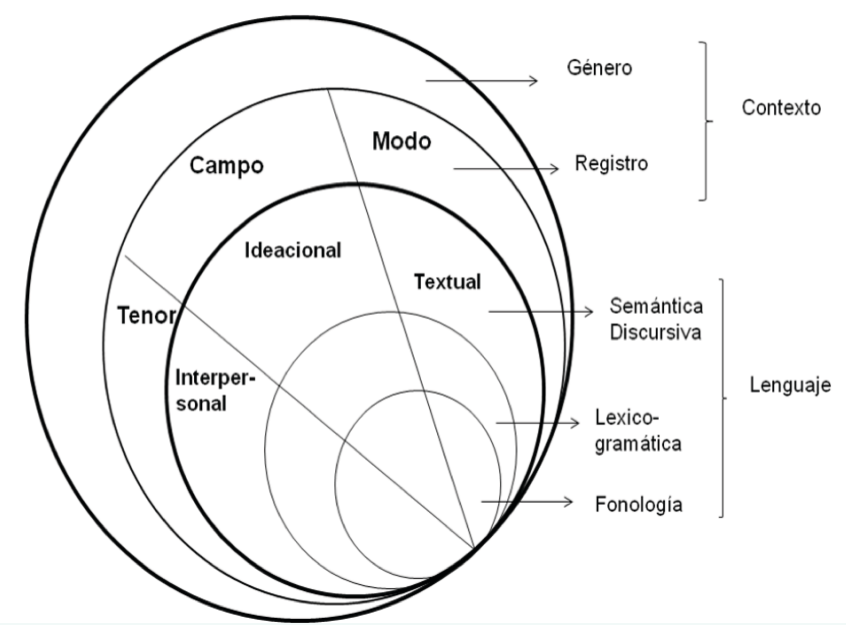

Figura 1 - Contexto y lenguaje estratificados (adaptado de Martin \& Rose 2007: 309).

En el Cuadro 1 se pueden observar las mismas relaciones, pero se explicitan los patrones semántico-discursivos y léxico-gramaticales que, como sistemas de opciones, están disponibles en el lenguaje para realizar significados. El género se encuentra más allá de las variables de registro, a fin de poder combinarlas libremente. En efecto, un mismo género puede variar en campo (un informe descriptivo puede aparecer en el contexto de las ciencias naturales o las sociales), en tenor (puede tratarse de una comunicación entre especialistas o de especialistas para legos, por ejemplo), y en modo (puede ser oral o escrito). Estas distintas variables de registro se manifiestan en el texto a través de la selección de recursos de los sistemas de opciones de significado que se agrupan por metafunción en el lenguaje en sus diferentes estratos. 
Cuadro 1 - Relaciones entre contexto y lenguaje: patrones semántico-discursivos y léxico-gramaticales (adaptado de Eggins \& Martin 2003).

\begin{tabular}{|c|c|c|c|c|}
\hline \multicolumn{2}{|c|}{ CONTEXTO } & \multicolumn{3}{|l|}{ LENGUAJE } \\
\hline \multirow{4}{*}{$\begin{array}{l}\mathbf{G} \\
\mathbf{E} \\
\mathbf{N} \\
\mathbf{E} \\
\mathbf{R} \\
\mathbf{O}\end{array}$} & $\begin{array}{l}\text { Variable de } \\
\text { Registro }\end{array}$ & $\begin{array}{l}\text { Tipo de } \\
\text { significado } \\
\text { en juego }\end{array}$ & $\begin{array}{l}\text { Patrones semántico- } \\
\text { discursivos }\end{array}$ & $\begin{array}{l}\text { Patrones léxico- } \\
\text { gramaticales }\end{array}$ \\
\hline & Campo & Ideacional & $\begin{array}{l}\text { * Ideación } \\
\text { * Conjunción }\end{array}$ & $\begin{array}{l}\text { * Transitividad (caso) } \\
\text { * Relaciones lógico- } \\
\text { semánticas (taxis) }\end{array}$ \\
\hline & Tenor & Interpersonal & $\begin{array}{l}\text { * Función del habla } \\
\text { * Negociación } \\
\text { * Valoración }\end{array}$ & $\begin{array}{l}\text { * Modo } \\
\text { * Modalidad } \\
\text { * Vocación }\end{array}$ \\
\hline & Modo & Textual & $\begin{array}{l}\text { * Identificación } \\
\text { * Periodicidad }\end{array}$ & $\begin{array}{l}* \text { Tema } \\
* \text { Flujo de la } \\
\text { Información }\end{array}$ \\
\hline
\end{tabular}

Es este complejo modelo el que provee suficientes elementos para sustentar el análisis de los textos que se presenta a los estudiantes y la reflexión acerca de cómo los recursos seleccionados para la realización del texto producen significado. El docente debe, sin embargo, hacer una selección de los recursos más relevantes para caracterizar los géneros bajo estudio y orientar a los estudiantes para comprender los significados que construyen. Para lograr este propósito, se necesita además una propuesta didáctica adecuada.

\subsection{La opción didáctica: una enseñanza de la lectura y la escritura basada en el concepto de género}

La propuesta didáctica adoptada para la enseñanza de la lectura y la escritura de textos académicos y profesionales a lo largo de las diferentes carreras universitarias es un modelo basado en los desarrollos de la Escuela de Sydney (Martin 1999; 2009b; Rose \& Martin 2012). Se trata de una didáctica explícita (Bernstein 1990), a través de la cual se pone en evidencia frente a los estudiantes qué acciones se llevan a cabo en el aula y con qué objetivos. La propuesta tiene en cuenta, además, el concepto de andamiaje, propuesto por primera vez por Wood, Bruner y Ross (1976), que consiste en el apoyo y guía que un profesor ofrece a los 
estudiantes en un proceso interactivo de construcción del aprendizaje que va más allá del ya adquirido por el grupo.

El modelo (Moyano 2007; 2013a) implica la enseñanza de la escritura a partir de la lectura y se propone también como enseñanza de contenidos disciplinares mediada por la lectura y la escritura. Se basa en la enseñanza de géneros y su realización lingüística e implica tres etapas: la Deconstrucción del género, el Diseño y Construcción de ejemplares genéricos y la Edición de los propios escritos. De estas etapas, la Edición no se encuentra presente en el modelo de la Escuela de Sydney, así como tampoco la subetapa Diseño, correspondiente a la etapa Construcción. Todos estos momentos están atravesados por la reflexión sobre el contexto y la negociación del campo y, en su puesta en práctica, se busca favorecer el desarrollo de la autonomía del estudiante tanto en la lectura como en la escritura de los textos que sus docentes les demandan, trabajando cada etapa primero de manera conjunta con el docente, luego en pequeños grupos y finalmente de manera individual (Figura 2).

De este modo, se busca que el estudiante haga un tránsito desde la heteronomía a la autonomía en las diferentes prácticas a llevar a cabo. Con este fin, en el trabajo conjunto se modelan, en interacción, los procesos de lectura y escritura como actividad, el control de los procesos cognitivos involucrados, la elección de opciones de género, discurso y recursos léxico-gramaticales en cada una de las etapas del modelo. En el trabajo en pequeños grupos, el docente asiste el trabajo de los estudiantes y retira gradualmente su andamiaje hasta el momento del trabajo autónomo, manteniendo, sin embargo, espacio para la consulta.

La Deconstrucción consiste en el trabajo analítico sobre un texto, instanciación del género que se busca enseñar. Como ya se anticipó, en ese trabajo se ponen en evidencia tanto la estructura esquemática del género como algunos rasgos discursivos que lo caracterizan y otros rasgos lingüísticos propios de la disciplina a la que el texto pertenece. Asimismo, es fundamental que los rasgos observados se entiendan como recursos para la producción de sentido: no basta con identificar elementos del texto sino que es necesario mostrar cómo se utilizan para la construcción de significados, ya sean ideacionales, interpersonales o textuales. 


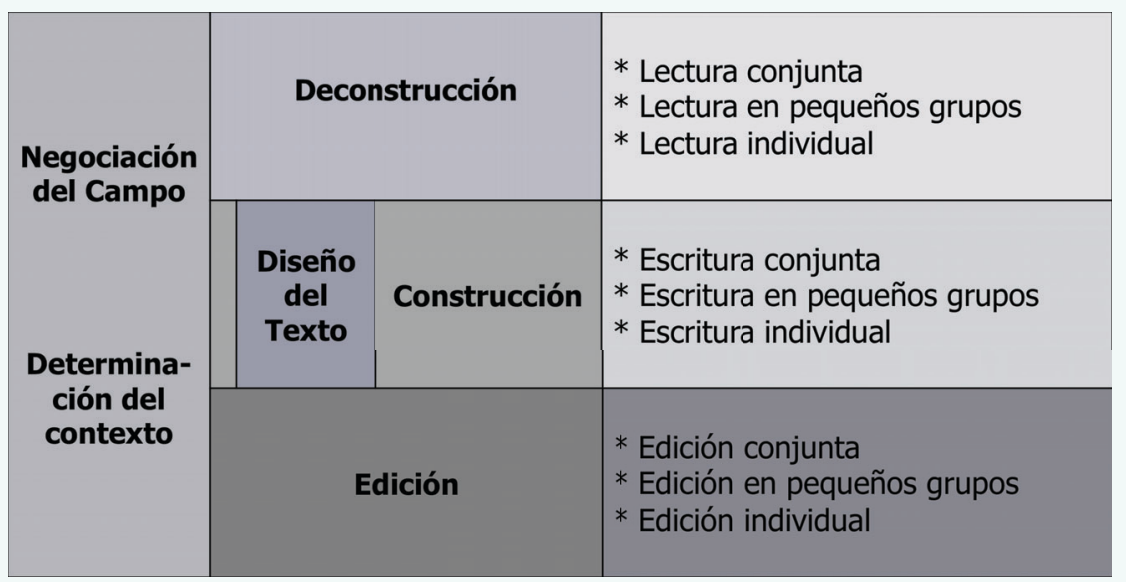

Figura 2 - Propuesta didáctica (Moyano 2007)

La intervención del docente de la materia en la que se trabaja es fundamental a la hora de reflexionar sobre los significados ideacionales para comprender cómo se construye campo en el texto, acudiendo a la bibliografía que los estudiantes han tenido que leer. Asimismo, la deconstrucción del tenor aporta información esencial que le permite al estudiante comprender cómo construye subjetividad el autor del texto y aprender recursos de valoración para aplicar posteriormente a sus producciones, a fin de responder a las demandas de la comunidad discursiva en la que se inserte. La identificación de recursos textuales, especialmente la Periodicidad (Martin \& Rose 2007; Moyano 2016), permiten orientar al estudiante hacia la estructuración del texto. En cuanto a la determinación del contexto, se trata no solo de identificar el género y su propósito sino de vincularlo con otros géneros del ámbito social al que pertenezca.

Difícilmente haya ocasión en el ámbito de la Universidad para trabajar el segundo momento del modelo: la Construcción conjunta de un texto como instancia del género estudiado. Sin embargo, es necesario dedicar un tiempo para reflexionar sobre su planificación y Diseño antes de que la escritura sea llevada a cabo de manera grupal o individual. En el caso de clases orientadas a la enseñanza de recursos como la metáfora gramatical (Halliday 1998) o los recursos de compromiso de la teoría de la valoración (Martin \& White 2005; Hood 2010; Moyano 2013b; 
2015), será necesario dedicar un momento de la clase para trabajar de manera conjunta la escritura.

Una etapa crítica de la propuesta didáctica es la Edición del propio texto. Obsérvese que no se habla de evaluación, corrección ni re-escritura, pues estos términos conllevan elementos semánticos negativos. El término "Edición", en cambio, sugiere la idea de texto como proceso, texto que se construye a lo largo de sucesivas modificaciones, proponiendo nuevas elecciones que mejoren la construcción del significado y contribuyan al logro del propósito perseguido. Esta etapa, que no se encuentra en el modelo de la Escuela de Sydney, surgió como propuesta para la enseñanza de un procedimiento que realizan los escritores expertos y que, en general, los docentes esperan que los estudiantes lleven a cabo sin más indicaciones que notas al margen de sus escritos. En la práctica docente, se ha observado que los estudiantes no necesariamente comprenden esas indicaciones y tienden a tomar una de las dos opciones siguientes: o aceptan las correcciones simplemente porque vienen de su profesor, sin entender de qué manera inciden en la producción de significado, o las ignoran, cuestionando la propuesta docente.

La Edición conjunta consiste en poner a consideración del grupo de estudiantes la producción de al menos uno de ellos, a fin de enseñarles cómo aplicar lo aprendido en la Deconstrucción a la revisión del texto para su Edición. Se espera que este trabajo se realice en interacción entre el docente y el grupo, de manera que los mismos estudiantes señalen los aciertos del texto así como los problemas que presenta, de modo de proponer para la discusión las modificaciones que consideran es necesario hacerle.

De la misma manera que en las etapas anteriores, lo que se observa en los textos que se editan es si han sido estructurados de acuerdo con lo esperado para el género y si se han utilizado los recursos discursivos y léxico-gramaticales observados en la Deconstrucción para la producción de significado. Asimismo, se espera que los estudiantes identifiquen zonas del texto que presenten problemas de construcción que deriven en la dificultad para su comprensión y puedan proponer alternativas para la resolución de estos problemas.

En este proceso, se va modificando o editando el texto hasta que logra su forma definitiva. Esta interacción es la que permite 
el modelaje de la actividad a través de lo que Rogoff (1994) llama "mente compartida": lo que se modela son las "discusiones" que el autor tendrá consigo mismo en el momento de editar su propio texto de manera autónoma.

Esta metodología ha mostrado producir resultados notables en la evolución de la escritura de los estudiantes. Moyano (2007), por ejemplo, muestra cómo estudiantes de un curso de escritura anterior a la carrera avanzan desde la producción de no-textos a la producción de textos adecuados a los géneros solicitados. Asimismo, se muestra a través de tablas de resultados cuantificados esa evolución, tanto en lo que hace a cada estudiante en sus textos como a dos grupos de estudiantes por rasgo evaluado. En otros trabajos (Giudice \& Moyano 2011; Moyano \& Giudice 2016a, por ejemplo), se muestran resultados cualitativos de la aplicación del modelo en el marco de dos diferentes programas de lectura y escritura académica a lo largo de la carrera.

\subsection{Diseño de gestión institucional}

Finalmente, para la instalación de un programa de lectura y escritura a lo largo de la carrera en una institución universitaria, es necesario un diseño de gestión institucional. Muchas veces, la práctica de asistencia en esta materia a los estudiantes se ha llevado a cabo individualmente, sin apoyo institucional (ver, por ejemplo, los casos presentados en UNLu (2001), la propuesta de Carlino (2005) o algunos de los trabajos publicados en Parodi (2010) o en Vázquez et al (2012)). Sin embargo, es importante tener en cuenta que, para que sea efectiva en términos de resultados en el desarrollo de habilidades de los estudiantes, la tarea de la lectura y la escritura en el nivel universitario no puede depender del esfuerzo individual de un docente o de grupos de ellos.

El modelo de programa que se propone en este trabajo contempla, en primer lugar, la negociación con autoridades académicas de diferente nivel según la estructura de poder de cada institución: Rectorado, Vicerrectorado, Secretaría Académica, Decanos de Facultades o cargos equivalentes, Directores de Carrera. Todos ellos tienen injerencia en diferentes aspectos de la cadena, que son relevantes a la hora de instalar acciones tendientes a la creación de un programa. 
En segundo lugar, es imprescindible la conformación de un equipo de trabajo integrado por profesores de Letras, que necesitarán especializarse en el sustento teórico del programa, el análisis de géneros académicos y profesionales, la propuesta didáctica seleccionada y los modos de interacción con los profesores de las materias específicas de las carreras. Esto requerirá formación en servicio, ya que una etapa de formación previa retardaría la puesta en marcha del programa una vez aceptado por las autoridades de la institución. Para que esto sea posible, es necesario seleccionar profesionales con interés en el área y que hayan tenido experiencia en enseñanza del discurso académico.

Una vez realizada la negociación con las autoridades de más alto nivel, que son las que autorizan la instalación del programa, se vuelve necesario trabajar con Decanos y Directores de carreras para la selección de las materias que van a participar de la propuesta. Los criterios a tener en cuenta para esta selección son al menos dos: en primer lugar, es importante que se elijan materias troncales, de manera de asegurar cierta regularidad de los estudiantes en el acceso a materias asociadas al programa; en segundo lugar, se considerará la disposición de los docentes a cargo de esas materias, pues contar con su acuerdo para llevar a cabo las actividades que se planifiquen es esencial, no solo para ello sino para asegurar que los estudiantes aceptarán la participación en el programa como parte de su formación obligatoria.

Luego de seleccionar las materias a participar, se asegurará desde la Coordinación la conformación de equipos que realicen la negociación entre pares de acuerdo con lo planteado por Moyano $(2009 ; 2010)$ y Moyano \& Giudice (2016b). Esta etapa del proceso de instalación es crítica, y de ella depende que los docentes del programa puedan contar con el tiempo necesario de clases más el apoyo de los profesores responsables de la materia en la que trabajen. Los integrantes de estos equipos tendrán roles diferenciados pero absolutamente necesarios en la enseñanza de la lectura y la escritura de géneros académicos o profesionales: los docentes de Letras trabajarán en la enseñanza de los géneros (su estructuración, organización discursiva y realización gramatical), mientras los docentes de las disciplinas específicas se ocuparán de la enseñanza y reflexión sobre el campo a trabajar y sobre el contexto en que los géneros circulan; ambos tipos de docentes trabajarán también complementariamente en las clases de Edición conjunta, haciendo aportes desde su especialidad. 
Todas estas actividades resultan, sin embargo, un gran desafío para la gestión institucional. Se presentan dificultades desde la perspectiva del estudiante, desde la del docente de la materia específica y desde las autoridades. Con respecto a los estudiantes, suelen recibir con agrado la idea de ser asistidos en el desarrollo de habilidades de lectura y escritura especializadas. Sin embargo, cuando el trabajo se inicia, presentan algunas resistencias debido a que el modo de implementación no les resulta familiar y les exige dedicación adicional. En relación con los profesores, pueden surgir inconvenientes relacionados con el hecho de que se les resta tiempo para el dictado teórico de su materia, además de que el descontento inicial de los estudiantes los hace dudar de la efectividad de la tarea. Por otra parte, para ellos también hay un incremento de actividades, ya que la negociación entre pares es un proceso recurrente que se añade a sus tareas habituales. Finalmente, estas situaciones redundan también en la satisfacción de las autoridades, pues la obtención de resultados no es tan inmediatamente visible como se desearía: instalar un programa en una carrera con buenos resultados puede demorar entre uno y tres años de trabajo intenso.

Estas dificultades pueden explicarse acudiendo al concepto de habitus de Bourdieu (1996; 1999): nuevas prácticas generan desconcierto pues se enfrentan con el habitus construido por estudiantes y docentes en relación con los modos de comportamiento y actuación en los procesos de enseñanza-aprendizaje en las aulas universitarias.

Tovillas (2010) explica que el concepto de habitus es un sistema de disposiciones durables y transferibles, aprendidas durante la infancia (habitus primario) y durante otras instancias de socialización posterior (habitus secundario), como por ejemplo la escolarización. Este sistema de disposiciones "le permiten al sujeto un conjunto de comportamientos y de actitudes al tiempo que circunscriben su margen de acción" (Tovillas 2010: 65). De esta manera, puede sostenerse que los actores de una universidad, como por ejemplo los estudiantes y los docentes, han adquirido un sistema de disposiciones que estructuran los comportamientos y actitudes alrededor de los procesos de aprendizaje. Dicho de manera sencilla, habría una manera de llevar adelante los procesos de enseñanza y aprendizaje que estos actores tienen incorporada. Una nueva metodología en el aula para el aprendizaje de la lectura y la escritura a fin de mejorar los aprendizajes 
de contenido chocaría de alguna manera con esas disposiciones, produciendo cierto desconcierto y hasta rechazo por no formar parte de ese sistema de disposiciones socialmente adquirido. Es que los habitus tienden a ser resistentes al cambio y marcan una continuidad en los comportamientos.

Sin embargo, el mismo Bourdieu ofrece una salida al problema: los habitus admiten modificaciones cuando se producen cambios de contexto para la produccion de esquema internos. Así, se pueden producir cambios en ellos, de manera que los individuos pueden modificar sus posturas y actitudes, reestructurar sus habitus en función de una nueva trayectoria social recorrida en las instituciones (Bourdieu 2006; Tovillas 2010).

De esta manera, puede pensarse que la clave para la resolución de problemas está en su anticipación. Si tanto la Coordinación como el equipo de docentes que integran el programa son conscientes de estas dificultades, deberán tener el mayor cuidado en los diferentes momentos de negociación, trabajo que requiere reconocer el lugar de autoridad de aquellos con los que se generan los intercambios, asegurarse que cada uno tenga su espacio de decisión mostrando flexibilidad en el diseño de aplicación sin desvirtuarlo, lograr que el conjunto de materias de cada carrera que se asocian al programa informe a los estudiantes de esta situación ya desde la incorporación de una leyenda en el programa de la asignatura. Esto generará nuevos contextos que permitan un cambio en la disposición de los sujetos, de manera de poder dar lugar a la creación de nuevos habitus, que permitan reconocer las nuevas prácticas como propias del ámbito social de la formación superior.

Aun así se generarán problemas que deberán ser sorteados hasta poder mostrar suficientes resultados positivos de la aplicación del programa. Por un lado, se necesita mostrar la evolución de los estudiantes en sus habilidades, así como también su valoración de las actividades realizadas y sus logros. Por otro, la satisfacción de los docentes de las materias específicas en cuanto a los aprendizajes de sus estudiantes tanto en cuanto a la lectura y la escritura como en relación a los contenidos de la materia. Asimismo, una satisfacción de ambos actores en relación con el vínculo con el docente asociado a la materia y con la Coordinación del programa resultan fundamentales. 


\section{Algunos resultados del PROLEA-UFLO}

El Programa de Lectura y Escritura Académicas de la Universidad de Flores se aprobó definitivamente en 2015, luego de dos años de una prueba piloto efectuada en la carrera de Psicología, en la Facultad de Psicología y Ciencias Sociales. A partir de 2015, se trabajó en la instalación del Programa en la carrera de Psicopedagogía, de la misma Facultad; las carreras de Profesorado de Educación Física y Licenciatura en Actividad Física y Deportes, de la Facultad de Actividad Física y Deportes; la carrera de Abogacía, en la Facultad de Derecho y las carreras de Ingeniería Ambiental y la Licenciatura en Seguridad e Higiene, de la Facultad de Ingeniería. Esto significa que, de las 6 Facultades de la Universidad, 4 se han asociado al Programa con sus carreras de dictado presencial, que en total suman 7 .

Por otra parte, el Programa se encuentra bajo la Dirección Académica, que se ocupa de la vinculación entre todos los programas o áreas de carácter transversal, como el Servicio de Orientación al Estudiante (SOE), el Programa de Acción Tutorial (PAT), y los créditos, como Idiomas y la capacitación en Informática.

Estos datos muestran que el Programa ha alcanzado un alto grado de inserción institucional, como logro de la gestión. Al momento de la producción de este artículo (diciembre de 2016), restan incorporar al Programa dos Facultades, la de Administración y la de Arquitectura y Diseño, trabajo que se realizará gradualmente a partir de 2017.

Por otra parte, se está logrando la integración del equipo docente del PROLEA, que cuenta hasta el momento con un profesor por cada Facultad asociada al Programa y una Coordinadora. Esto se logra de dos maneras. Por un lado, a través de la realización periódica de reuniones de discusión de las prácticas llevadas a cabo, tanto en relación con la negociación entre pares como con la enseñanza de la lectura y la escritura en cada una de las materias asociadas. Por otro lado, los integrantes del equipo se agrupan en torno a la investigación, que incluye la revisión bibliográfica para la formación en servicio, el análisis de algunas de las prácticas llevadas a cabo (Moyano \& Acebal 2016a; Moyano \& Giudice 2016a), el seguimiento de la evolución de los textos producidos por los estudiantes (Giudice, en prensa; Moyano 
\& Acebal 2016b) así como de la relación con los docentes de materias específicas en la negociación entre pares (Moyano \& Giudice 2016b) y los cambios en la gestión de las carreras (Moyano \& Godoy 2015). A medida que los docentes se fueron integrando al Programa, se fueron también integrando gradualmente al equipo de investigación, cada uno con diferentes actividades, de acuerdo con el avance de manejo de la teoría.

En cuanto a la evolución de los docentes de las materias específicas en términos de aceptación del trabajo conjunto, se han notado diferentes grados de involucramiento. Sin embargo, a medida que pasa el tiempo, se van consolidando los equipos de pares que van avanzando en los acuerdos establecidos en la negociación. Esto se observa en la manifestación de los docentes de las materias específicas acerca de su satisfacción con respecto a los logros obtenidos. Por el momento, estas manifestaciones se han obtenido como comunicaciones personales a través del envío de correos electrónicos o en reuniones de trabajo de evaluación de la propuesta, sin que se hayan formalizado estos encuentros para el registro de datos para analizar en investigación. Para llegar a esta etapa es necesario también un proceso que conduzca a la aceptación de la grabación de entrevistas y a la realización de grupos focales, técnicas que están previstas para avanzar con la investigación. No obstante eso, en dos de las carreras involucradas se han tomado encuestas a los docentes, que están actualmente en procesamiento.

Finalmente, es importante destacar los logros en relación con los estudiantes. A través del análisis de trabajos producidos por ellos, se ha observado un avance en las habilidades de lectura y escritura (cf. por ejemplo, Giudice \& Moyano 2011; Moyano \& Giudice 2016a; Giudice en prensa, Moyano \& Acebal 2016b). En este momento, se está avanzando en continuar con estudios de este tipo, que permiten mostrar los logros de los estudiantes, que redundan en el aprendizaje de contenidos por reelaboración (Bereiter \& Scardamaglia 1987; Halliday 1993a). Por otra parte, se registra un mayor compromiso de los estudiantes en su partcipación de las actividades del Programa, de manera que se puede afirmar que esta práctica ha comenzado a naturalizarse, cambiando el habitus. Esto puede observarse a partir de algunos correos electrónicos de agradecimiento enviados por los 
estudiantes a los docentes PROLEA así como de solicitudes de mayor inserción en la carrera.

\section{Consideraciones finales}

El presente trabajo tiene por objetivo presentar como opción para la enseñanza de la lectura y la escritura de textos académicos y profesionales la instalación de programas a lo largo de la carrera. Así, se presentó el problema que genera esta necesidad, mostrando que no se trata de una deficiencia en la formación de los estudiantes sino de su ingreso a una nueva comunidad discursiva, un nuevo ámbito de actividad social que requiere un manejo del discurso especializado así como de géneros que hasta ese momento habían quedado fuera de su esfera de actuación. Esta interpretación del problema pone en evidencia que son todos los estudiantes los que necesitan de la formación en esta área, aunque algunos estén en mejores condiciones que otros según su experiencia educativa los haya colocado o no en la zona de desarrollo próximo de estos aprendizajes.

Como caso para ejemplificar la organización de un programa de lectura y escritura se presentó el PROLEA-UFLO, que tiene como antecedente inmediato el PRODEAC instalado en la UNGS.

A partir de allí, se introdujeron los requerimientos teórico metodológicos para la instalación de un programa de este tipo, así como las demandas de gestión. Se abordó entonces la posición tomada con respecto a la teoría de aprendizaje a través del lenguaje enmarcada en la Lingüística Sistémico-Funcional, marco general en el que se basa la propuesta. Inmediatamente se desarrollaron los conceptos básicos de esta teoría lingüística que resultan esenciales para abordar el trabajo de enseñanza en juego. Asimismo, se presentó el modelo didáctico que, desarrollado a partir del de la Escuela de Sydney, se implementa para la ejecución del programa. Finalmente, se abordaron los lineamientos de gestión desarrollados para la institucionalización de un programa de escritura y, para cerrar, algunos resultados obtenidos en el corto tiempo de instalación del PROLEA-UFLO.

Llevar a cabo programas como el que aquí se propone presenta ciertas dificultades, como se sugiere en este trabajo. Sin embargo, los 
resultados de aplicación que se van perfilando en el PROLEA-UFLO tanto como otros observados en el PRODEAC (cf. los trabajos citados en Moyano 2010) sugieren que la opción es válida para los objetivos que se persiguen. En efecto, como se señaló antes, presenta ventajas con respecto a los cursos o talleres paralelos a las materias específicas de las carreras en las que se instalan así como al trabajo limitado que pueden llevar a cabo los docentes de esas materias específicas, que tienen otras especialidades. La propuesta de un trabajo interdisciplinario, en el que cada docente pueda hacer aquello para lo que se ha preparado, parece una opción más adecuada.

Por otra parte, es toda la institución la que se ve modificada por esta práctica, de manera que el compromiso institucional contribuye a la obtención de logros significativos con respecto a todos los actores involucrados.

Finalmente, el espacio de investigación asociado al Programa permite un monitoreo permanente de todas las actividades que éste demanda, de manera que se pueden ir generando modificaciones para optimizar la práctica.

A manera de cierre, es importante señalar que este tipo de programas muestran la asunción de responsabilidad de las instituciones que los adoptan en relación con la formación de los estudiantes, a fin de asistirlos para que logren avanzar en la carrera que han elegido hasta obtener su graduación y en asegurarles preparación para las actividades de lectura y escritura que deban realizar en el marco del ejercicio de sus profesiones, independientemente de la procedencia socio-cultural de los estudiantes. Seguramente no será ésta la única variable para lograr que los estudiantes completen sus estudios universitarios. Pero, sin lugar a dudas, es una variable relevante: parafraseando a Martin (1993), si los estudiantes no logran manejar los recursos necesarios para la producción de significados especializados, quedarán fuera de los procesos en los que desean involucrarse en la universidad, no solo en lo que hace a lo académico sino también a su participación política y social.

Recebido em: 03/03/2017

Aprovado em: 27/04/2017

E-mail: estelainesmoyano@gmail.com 


\section{Referencias bibliográficas}

Ameijde, D.; Murga, M.; Padilla, C. y Douglas, S. 2000. Conceptualizaciones sobre el saber lingüístico en el discurso estudiantil. Actas del Congreso Nacional de Lingüística, Mar del Plata, Sociedad Argentina de Lingüística (publicación electrónica).

Bereiter, Carl y Scardamalia, Marlene. 1987. The psychology of written composition. Hillsdale, N.J: Erlbaum.

Bernstein, Basil. 1990. Class, Codes and Control, Vol 4. The structuring of Pedagogic Discourse. London: Routledge.

1999. Vertical and Horizontal Discourse: an essay. British Journal of Sociology of Education, Vol 20 (2): 157-173.

Bourdieu, Pierre. 1996. Cosas Dichas. Barcelona: Gedisa. 1999. Meditaciones Pascalianas. Barcelona: Anagrama.

.2006. Argelia 60. Estructuras económicas y estructuras temporales. Buenos Aires: Siglo XXI.

CARLINo, Paula. 2005. Escribir, leer y aprender en la universidad. Una introducción a la afabetización académica. Buenos Aires: FCE.

Cubo de Severino, L. 2002. Evaluación de estrategias retóricas en la comprensión de manuales universitario. RILL, No15. Tucumán, INSIL.

De Lelia, Cayetano y Ezcurra, Ana María. (Dir.). 1994. Competencias cognitivas de alumnos del penúltimo año de educación media. Instituto de Estudio Acción Social. Investigación destinada a la Universidad Nacional de General Sarmiento.

EgGins, Susan \& Martin, James. 2003. El contexto como género: una perspectiva lingüístico-funcional. Revista Signos, 36(54): 185-205.

EzcurRa, Ana María. (Dir.). 1995. Competencias cognitivas de alumnos de primer ingreso universitario potencial (en actividades de comprensión y producción de textos de tipo expositivo y argumentativo de género académico). Investigación de la Unidad Pedagógica Universitaria, Universidad Nacional de General Sarmiento. Investigadores del área de Lengua: Profs. S. Nogueira, M. C. Pereira.

2011. Igualdad en educación superior. Un desafio mundial. Los Polvorines: Universidad Nacional de General Sarmiento.

GiUdicE, Jacqueline. En prensa. Enseñanza de la reseña bibliográfica a través de un programa de lectura y escritura académica. En Homenaje a Elvira Arnoux. Estudios de análisis de discurso, glotopolítica y pedagogía de la lectura y la escritura. Buenos Aires: Oficina de Publicaciones de la Facultad de Filosofía y Letras de la UBA (OPFyL). 
\& Moyano, E. 2011. Apropiación del discurso de la economía: Análisis evolutivo de un caso. En: Barbara, L. \& E. Moyano (Eds.). Textos y lenguaje académico. Exploraciones sistémico-funcionales en portugués y español. Los Polvorines/San Pablo: UNGS-PUCSP. p. 91-112.

Halliday, Michael. 1964 [2003]. Syntax and the consumer. En: On Language and Linguistics. Collected Works of M.A.K. Halliday, Vol.

3. Ed by Jonathan Webster. London: Continuum. p. 36-49.

1978 [1982]. El lenguaje como semiótica social [Traducción: Jorge Ferreiro Santana]. México: FCE.

. 1980 [2004]. Three aspects of children's language development: learning language, learning trhough language, learning about language. En: The Language of Early Childhood. Collected Works of M.A.K. Halliday, Vol. 4. Ed by Jonathan Webster. London: Continuum. p. 308-326.

. 1993a Towards a Language-based theory of learning. Linguistics and Education, 5, p. 93-116.

1993b. On the language of Physical Science. En: Michael Halliday \& James Martin: Writing science: Literacy and discursive power. Pittsburgh: University of Pittsburgh Press. p. 54-68. . 1995 [2005]. Computing Meanings: Some Reflections on Past Experience and Present Prospects. En: Computational and Quantitative Studies. Collected Works of M.A.K. Halliday, Vol 6, Ed. By Jonathan J. Webster. London: Continuum. p. 239-267.

1998. Things and Relations. Regrammaticising experience as technical knowledge. En: Martin, James \& Veel, Robert. Reading Science. Critical and Functional Perspectives on Discourses of Science. London: Routledge. p. 185-235.

\& Martin, James. 1993. Writing science: Literacy and discursive power. Pittsburgh: University of Pittsburgh Press.

\& Matthiessen, Christian. 1999. Construing experience through meaning: a language-based approach to cognition. London: Cassell.

Hyland, Ken. 2002. Teaching and Researching Writing. London: Longman.

Hood, Susan. 2010. Appraising Research. Evaluation in Academic Writing. London: Palgrave Macmillan.

\& Martin, James. 2005. Invocación de actitudes: El juego de la gradación de la valoración en el discurso. Revista Signos, 38 (58): 195-220. 
Korner, H.; McInNEs, D. \& Rose, D. 2007. Science literacy. NSW: NSW AMES.

Martin, James. 1985. Process and text: two aspects of human semiosis. En: Benson, J.D. \& Greaves, W. S. (Eds.). Systemic Perspectives on Discourse: selected theoretical papers from the 9th International Systemic Workshop. Norwood, N.J.: Ablex. p. 248-274.

1992. English Text: System and Structure. Amsterdam: Benjamins.

1993. A contextual theory of language. En: Cope, W. \& Kalantzis, M. [Eds.]. The Powers of Literacy: a genre approach to teaching literacy. London: Falmer (Critical Perspectives on Literacy and Education) \& Pittsburg: University of Pittsburg Press (Pittsburg Series in Composition, Literacy, and Culture). p. 116-136.

1994. Macro-genres: the ecology of the page. Network 21: $29-52$

. 1997. Analyzing genre: functional parameters. En: CHRISTIE, F. \& MARTin, J.R. (Ed.). Genre and institutions: Social processes in the workplace and school. London: Continuum. p. 3-39.

1999b. Mentoring semogenesis: 'genre-based' literacy pedagogy.

En: Christie, F. (Ed.). Pedagogy and the Shaping of Consciousness: linguistic and social processes. London: Cassell (Open Linguistics Series). p. 123-155.

.2000. Grammar meets genre - reflections on the 'Sydney School'. Arts: the journal of the Sydney University Arts Association 22: 47-95.

2001. A context for genre: modelling social processes in functional linguistics. En: Devilliers, J \& Stainton, R. (Eds.). Communication in Linguistics: papers in honour of Michael Gregory. Toronto: GREF (Theoria Series 10), p. 287-328.

. 2009a. Boomer dreaming: the texture of recolonisation in a lifestyle magazine. En: Forey, G. \& Thompson, G. (Eds.). Text-type and Texture, London, UK: Equinox Publishing. p. 252-284.

2009b. Genre and language learning: A social semiotic perspective.

Linguistics and Education 20: 10-21.

.2014. Evolving systemic functional linguistics: beyond the clause.

Functional Linguistics, 1:3.

.2016. One of three traditions: genre, functional linguistics and the 'Sydney School'. En: Artemeva, N. (Ed.). Papers arising form the Rethinking Genre conference in Ottawa in June 2012. 
\& DAVID, Rose. 2007. Working with Discourse. Meaning Beyond the Clause. 2nd Ed. London: Continuum. \& 2008. Genre Relations. Mapping culture. London: Equinox.

\& RotheRY, Joan. 1993. Grammar: making meaning in writing. En: W Cope \& M Kalantzis [Eds.]. The Powers of Literacy: a genre approach to teaching literacy. London: Falmer (Critical Perspectives on Literacy and Education) \& Pittsburg: University of Pittsburg Press (Pittsburg Series in Composition, Literacy, and Culture). p. 137-153.

\& Veel, Robert (Eds.). 1998. Reading Science. Critical and Functional Perspectives on Discourses of Science. London: Routlege.

\& White, Peter. 2005. The language of evaluation. Appraisal in English. London: Palgrave.

Moyano, Estela Inés. 2003. Evaluación Diagnóstica Sumativa del Taller de Lecto-escritura del curso de aprestamiento Universitario de la UNGS, Informe Final de Resultados, Publicación interna Secretaría Académica, IDH-UNGS, Bs.As., Argentina.

. 2004. La Escritura Académica: Una Tarea Interdisciplinaria a lo largo de la Curricula Universitaria. Revista Texturas 4 (4), 109-120. . 2007. Enseñanza de Habilidades Discursivas en Español en Contexto Pre-universitario: Una Aproximación desde la LSF. Revista Signos 40(65), 573-608.

.2009. Negotiating Genre: Lecturer's Awareness in Genre Across the Curriculum Project at the University Level. In: BAzerman, Ch.; BoninI, A. \& Figueiredo, D. Genre in a Changing World. Perspectives on Writing. p. 449-464. Fort Collins, Colorado: The WAC Clearinghouse and Parlor Press.

. 2010. Escritura Académica a lo largo de la Carrera: Un Programa Institucional. Revista Signos, 43(74), 465-488.

.2013a. Una didáctica de las ciencias basada en los géneros textuales: acceso a las disciplinas a través de la apropiación de su discurso. En: Moyano, E.I. (Coord.). Aprender ciencias y humanidades: una cuestión de lectura y escritura. Aportes para la construcción de un programa de inclusión social a través de la educación lingüistica. Los Polvorines: UNGS. p. 109-155.

. 2013b. Proyección congruente y metafórica en las discusiones de artículos científicos de tres disciplinas en español. En: VIAN JR., O. 
\& Caltabiano, C. (Orgs.). Lingua(gem) e suas múltiplas faces. San Pablo: Mercado de Letras. p. 109-133.

2015. Patrones de Realización de la Proyección en la Discusión de Artículos de Investigación Producidos en Español en Dos Disciplinas. Revista D.E.L.T.A. 31(1), 143-183.

.2016. Theme in English and Spanish: Different means of realization for the same textual function. En: Clark, B. \& Arús HitA, J. (Eds.). The dinamicity of communication below, around and above the clause. Special Issue of English Text Construction 9 (1): 190-220.

\& Acebal, Martín. 2016a. La infografía como recurso mediador en la enseñanza de habilidades de lectura y escritura académica. Ponencia presentada en las II Jornadas Nacionales y Regionales de Lingüística Sistémico-Funcional, Facultad de Humanidades y Ciencias (Universidad Nacional del Litoral), Santa Fe (Argentina), 4 y 5 de agosto de 2016. $\&$ 2016b. Adecuación de un género a la estructura de conocimiento de las Ciencias Sociales en la enseñanza de habilidades de lectura y escritura académicas. Ponencia presentada en el XII Congreso la Asociación de Lingüística Sistémico-Funcional de América Latina (ALSFAL), Universidad del Norte, Barranquillas (Colombia), 5-9 de septiembre de 2016.

\& GiUdiCE, Jacqueline. 2016a. Un Programa de Lectura y Escritura Universitario: Lineamientos Teóricos, Características y Resultados de Aplicación. Revista Grafía, 13 (1), enero a junio 2016.

$\&$ . 2016b. Negotiation Between Professional Peers: Critical Strategy for a Reading and Writing Program at the University Level. En: Bazerman, Ch. \& Moritz, M.E. Special Issue Higher Education Writing Studies in Latin America, Ilha do Desterro, 69: 3, JanuaryJune 2016.

\& Godoy, Marcelo. 2015. Desafíos de la gestión académica de un programa de escritura a lo largo de la carrera. XXII Congreso Internacional de Educación y Aprendizaje. Madrid, 9 al 11 de julio.

\& Natale, Lucía. 2012. Teaching Academic Literacy across the University Curriculum as Institutional Policy. The case of the Universidad Nacional de General Sarmiento (Argentina). In: ThaIss, Ch,; Bräuer, G.; Carlino, P.; Ganobcsik-Williams, L. \& Sinha, A. Writing Programs Worldwide: Profiles of Academic Writing in Many Places. Perspectives on Writing, pp. 23-34. Fort Collins, Colorado: The WAC Clearinghouse and Parlor Press. 
PAdilla, Constanza. 2007. ¿Enseñar a argumentar en la universidad? ¿Por qué y para qué? En: Actas Primeras Jornadas Latinoamericanas de Lectura y Escritura. Lecturas y escrituras críticas: perspectivas múltiples. Facultad de Filosofía y Letras de la Universidad Nacional de Tucumán. Disponible en: http://www.filo.unt.edu.ar/jorn_unesco/ jorn_unesco_cd.htm.

PAINTER, Claire. 1986. The rol of interaction in learning to speak and learning to write. En: C. Painter \& J.R. Martin (Eds.). Writing to mean: Teaching genres across the curriculum. Sydney: ALAA Occasional Papers 9. p. 62-97.

PARODI, Giovani. (Ed.). 2010. Alfabetización académica y profesional en el Siglo XXI: Leer y escribir desde las disciplinas. Santiago de Chile: Ariel.

Pereira, María Cecilia y di Stefano, Mariana. 2001. Diseño curricular del Taller de Lectura y Escritura universitario. La lectura y la escritura como procesos y como prácticas. Ponencia presentada en el XIX ${ }^{\circ}$ Congreso Asociación Española de Lingüística Aplicada (AESLA), organizado por la Universidad de León (España).

Rogoff, Barbara. 1994. Los tres planos de la actividad sociocultural: Apropiación participativa, participación guiada y aprendizaje. En: J.V. Wertsch., P. del Río \& A. Álvarez (Eds.). La mente sociocultural. Aproximaciones teóricas y aplicadas. Madrid: Fundación Infancia y Aprendizaje. p. 111-128.

Rose, D. \& Martin, J.R. 2012. Learning to Write, Reading to Learn. Genre, Knowledge and Pedagogy in the Sydney School. UK: Equinox.

Tovillas, Pablo. 2010. Bourdieu. Una introducción. Buenos Aires: Quadrata.

UNLu. 2001. La Lectura y Escritura como prácticas académicas universitarias. Departamento de Educación Luján, Bs. As., Argentina. Disponible en: http://www.unlu.edu.ar/ redecom/borrador.htm.

Uslenghi, M; Padilla, C. \& Sigstad, M. (Eds.). 2002. Textos de especialidad: Problemas y propuestas para la Universidad. RILL $\mathrm{N}^{\circ} 15$, Tucumán: INSIL, Facultad de Filosofía y Letras, Universidad Nacional de Tucumán.

VÁzquez, A.; JАKob, I.; Novo, M.C. \& Pelizza, L. (Comp.). 2012. Lectura, escritura y aprendizaje disciplinar. Río IV: UniRío Editora, Universidad Nacional de Río Cuarto.

VeEL, Robert. 1997. Learning how to mean - scientiffically speaking. Apprenticeship into scientific discourse in the secondary school. En: Christie, Frances. \& James Martin (Eds.). Genre and institutions. 
Social proceses in the workplace and school. London: Contiuum. p. 161-195.

Vigotsky, Lev. 1998. Pensamiento y lenguaje. Buenos Aires: Fausto.

Wignell, Peter. 2007. On the Discourse of Social Science. Australia: Charles Darwin University Press.

Wood, D.; Bruner, J. \& Ross, G. 1976. The Role of Tutoring in Problem Solving. Journal of Child Psychology and Psychiatry 17, 89-100.

ZaLBA, Estela. 2002. La palabra interdicta: problemas de producción discursiva en estudiantes universitario. Ponencia presentada en el Simposio Internacional Lectura y escritura: nuevos desafios, Facultad de Ciencias Políticas y Sociales \& Facultad de Filosofía y Letras de la Universidad Nacional de Cuyo. Mendoza, mayo de 2002. 